\title{
ESTUDO ETNOBOTÂNICO COM CRIANÇAS E ADOLESCENTES NA COMUNIDADE SÃO FRANCISCO, CABACEIRAS (PB)
}

\author{
Jaciara Bomfim dos Santos 1 \\ Thamires Kelly Nunes Carvalho ${ }^{2}$ \\ Ezequiel da Costa Ferreira ${ }^{3}$ \\ Suellen da Silva Santos ${ }^{4}$ \\ Camilla Marques de Lucena 5 \\ José Ribamar Farias Lima ${ }^{6}$ \\ Guilherme Muniz Nunes ${ }^{7}$ \\ Reinaldo Farias Paiva de Lucena ${ }^{8}$
}

\begin{abstract}
Resumo: A pesquisa foi realizada na comunidade São Francisco em Cabaceiras com objetivo de realizar um estudo etnobotânico com crianças e adolescentes. A coleta de dados foi realizada através de entrevistas semiestruturadas e lista livre. Os jovens caracterizaram cada espécie citada de acordo com a sua relação, informação sobre o uso e conhecimento pessoal, mencionando diversas categorias utilitárias. Dentre as plantas mais citadas, destacaram-se, Opuntia ficus indica L. Mill e Prosopis juliflora (SW). DC. Baseando-se na diversidade de plantas que foram mencionadas, a pesquisa foi de grande relevância. Enfatiza-se a necessidade na realização de outras pesquisas dessa natureza, sugerindo a adoção de métodos e perspectivas da educação ambiental, podendo a mesma ser utilizada no desenvolvimento educacional das crianças e adolescentes.
\end{abstract}

Palavras chave: População Tradicional; Semiárido; Educação.

\footnotetext{
${ }^{1}$ UFPB. CCEN. DSE. E-mail: janacinta.nogueira@gmail.com

2 UFPB. CCEN. DSE. E-mail: carvalhotkn@gmail.com

3 UFPB. CCEN. DSE. E-mail: ezequielcostaf@gmail.com

4 UFPB. CCEN. DSE. E-mail: suellenbiologa.ss@gmail.com

5 UFPB. CCEN. DSE. E-mail: camillamlucena@gmail.com

6 UFPB. CCEN. DSE. E-mail: ribamarfl@gmail.com

${ }^{7}$ UFPB. CCEN. DSE. E-mail: guilhermemnunes@hotmail.com

8 UFPB. CCEN. DSE. E-mail: rlucena@dse.ufpb.br
}

Revbea, São Paulo, V. 13, № 4: 390-407, 2018. 


\section{Introdução}

O dinamismo da modernidade agrega novos hábitos às populações locais e, por consequência, conduzem a perda e/ou transformações de antigas práticas tradicionais. Este cenário também é contextualizado pelo aumento da extração dos recursos naturais devido ao aumento da população e/ou da entrada destas na economia de mercado (AMOROZO, 2002). Podendo influenciar na pressão ambiental dessas populações e afunilar 0 processo de transmissão do conhecimento, tais mudanças atuais costumam ser motivo de preocupação, uma vez que podem se constituir em mecanismos de erosão dos conhecimentos e das espécies que fazem a riqueza da região e participam do manejo da diversidade biológica e cultural (EMPERAIRE et al., 2008).

Devido à demanda global a favor da proteção da natureza, juntamente com o crescimento de correntes ambientalistas detentoras de perspectivas diferentes da preservacionista, as populações tradicionais passaram a ser consideradas importantes como atores responsáveis pela proteção do ambiente natural no qual estão inseridas (PEREIRA; DIEGUES, 2010). Neste contexto sobre as mudanças ambientais, culturais e numa perspectiva conservacionista, Iturra (1993) afirma que todo o grupo social precisa transmitir a sua experiência acumulada no tempo à geração seguinte, como condição da sua continuidade histórica. $O$ fato de os membros individuais do grupo estar sempre a renovar-se, seja pela morte, seja pelo nascimento, dinamiza-se a necessidade de que essa experiência acumulada, que se denomina saber e existe fora do tempo individual, fique organizada numa memória que permaneça no tempo histórico.

Esses conhecimentos tendem a se acumular, de geração em geração e são transmitidos às crianças por práticas rotineiras, que naturalmente são inseridos a respeito do meio ambiente, uso, exploração e importância das espécies vegetais, sejam elas inseridas e ou nativas (MCCARTER; GAVIN 2011). Ressalta-se ainda, que o processo educativo é o comportamento que mais marca o cotidiano das nossas vidas, e é o mais cotidiano dos processos que orienta o nosso agir, seja como ensino, seja como aprendizagem, procurando sistematizar o conjunto do dia-a-dia de todos os seres humanos de diversas idades que coexistem.

Registrar como as populações tradicionais no seu cotidiano repassam o conhecimento para os mais jovens integrantes da comunidade pode servir de fonte para ações que busquem a integração cultural desses jovens com o ambiente e conservação do mesmo, além disso, estudos envolvendo os mais jovens podem ser importantes para avaliar se os conhecimentos sobre o uso de plantas e conservação da natureza estão sendo repassados internamente nas comunidades, no pressuposto de uma "etnoeducação" (BORTOLOTTO; GUARIMNETO 1998).

A etnobotânica contribui para o registro do saber local sobre a utilização dos 
recursos vegetais, visto que a mesma busca entender a dinâmica da relação pessoas/plantas (OLIVEIRA et al. 2009). Albuquerque (2005) cita que todas as ciências que se ocupam de investigar essa relação estão preocupadas em registrar e conhecer as estratégias e conhecimentos dos povos locais, procurando também usar essa informação em benefício dessas próprias pessoas.

Segundo Albuquerque (2005), a etnobotânica, possibilita desvendar formas específicas de manejo dos recursos naturais em cada ecossistema, bem como descobrir quais plantas apresentam um alto grau de importância, sendo este, reflexo de suas potencialidades, e aliado a estes fatores, propõe a elaboração de estratégias que garantam a conservação da diversidade local. Alguns estudos já abordam esta perspectiva da etnobotânica especificamente com crianças, a exemplo de García (2006), Freitas et al. (2011) e Acosta et al. (2015).

Em ambientes que apresentem uso intenso dos recursos naturais, como é o caso das populações existentes em ambientes rurais da Caatinga, registrar os conhecimentos locais, segundo Albuquerque e Andrade et al. (2002), é um passo a ser dado em busca da sua conservação. Quando se fala na Caatinga, muitos a relacionam a um ambiente homogêneo e pobre em biodiversidade, mas na verdade, grande parte do patrimônio biológico dessa região não é observada em nenhum outro lugar do mundo, senão, no Nordeste do Brasil (SILVA et al. 2017). Ocupando uma área de $912,529 \mathrm{Km}^{2}$, a Caatinga apresenta-se bastante heterogênea, pela presença em especial, de espécies vegetais endêmicas, em sua maioria de porte arbustivo e arbóreo que possuem estratégias de adaptação as condições extremas de clima e solo, como é o caso das regiões semiáridas (SILVA et al., 2017).

Freitas e Ribeiro (2007), afirmam que na infância começa a se formar a personalidade de uma pessoa e o modelo inicial formado é, diversas vezes, revisado, consolidado e estabelecido novamente. Portanto, o comportamento de um indivíduo adulto e a sua postura, inclusive em relação ao meio ambiente, pode ter sua origem na infância. Investigar como se dá essa relação desde a infância, parece ser uma importante iniciativa para a conservação das espécies, numa expectativa de prever o manejo local do ambiente e a exploração dos recursos naturais.

Uma vez que essa temática de pesquisa, realizada com crianças e adolescentes, apesar de ainda pouco utilizada, vem despertando interesse na comunidade acadêmica pela importância em se observar como a consciência ecológica tradicional é formada e repassada entre adultos e crianças e entre as próprias crianças, servindo como instrumento eficaz para a conservação cultural e ambiental de diversas comunidades, assim como pode-se aproveitar em ações de educação ambiental formal e não-formal. Envolvida nesse contexto ambiental e cultural, esta pesquisa teve como objetivo realizar um estudo etnobotânico com crianças e adolescentes na comunidade São Francisco, em Cabaceiras, Paraíba. 


\section{Materiais e métodos}

\section{Área de Estudo}

O trabalho foi realizado na comunidade São Francisco, zona rural do município de Cabaceiras-Paraíba, Nordeste do Brasil, localizado na mesorregião da Borborema e microrregião do Cariri Oriental, distando $66,7 \mathrm{Km}$ da cidade de Campina Grande (polo regional) e $180 \mathrm{~km}$ da capital do Estado, João Pessoa (Figura 1). Área localizada na unidade geoambiental do Planalto da Borborema e de acordo com o IBGE (2010), apresenta população estimada em 5.035 habitantes, distribuídos em uma área territorial de $453 \mathrm{~km}^{2}$, o que dá uma densidade demográfica de 11,12 hab./km² (Figura 1).

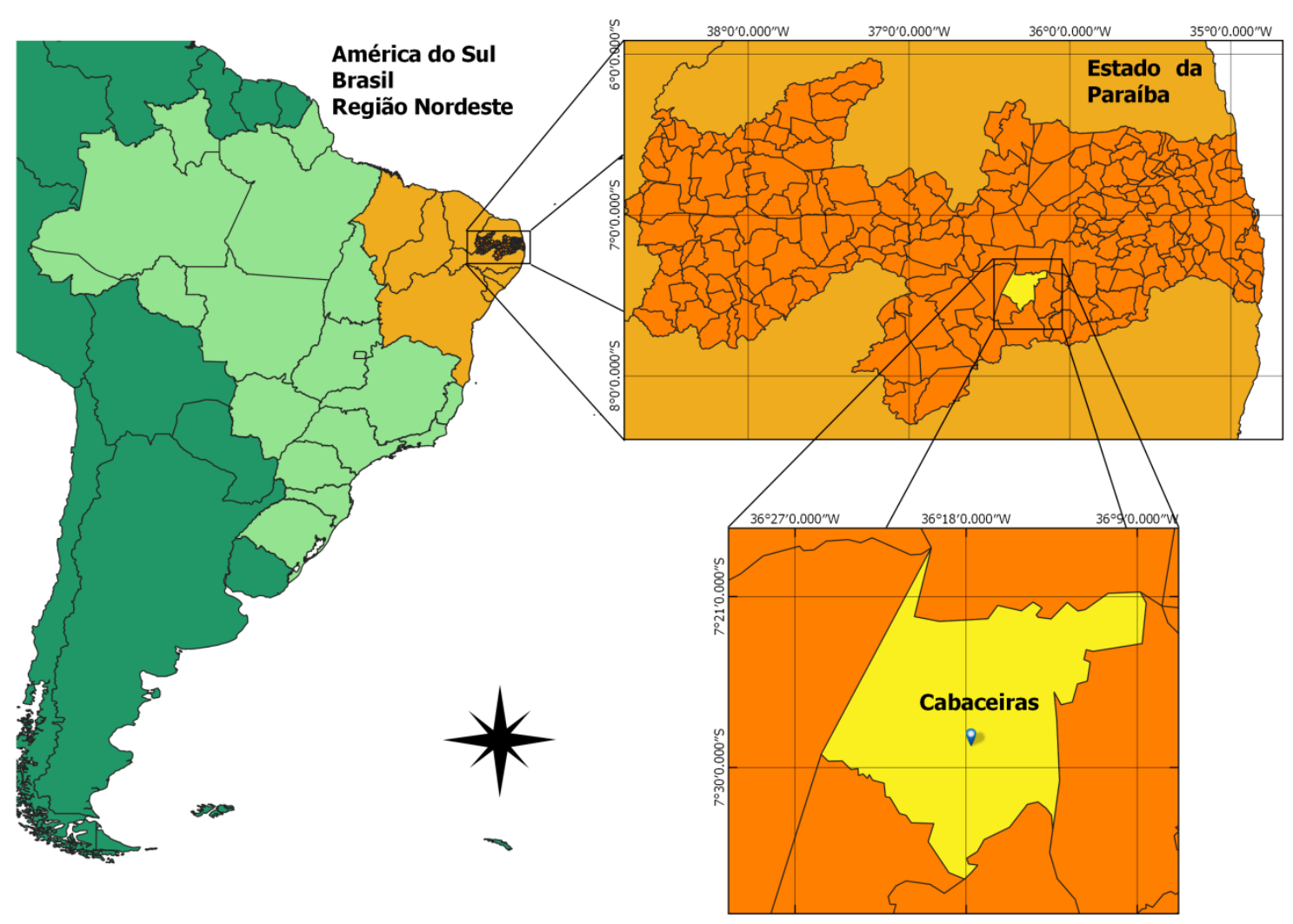

Figura 1: Localização do município de Cabaceiras, estado da Paraíba, Nordeste do Brasil.

O clima, segundo Köppen, é BSh (semiárido quente), com temperatura média anual superior a $20^{\circ} \mathrm{C}$, possuindo o menor índice pluviométrico do Brasil, menos de $263 \mathrm{~mm}$ de chuva durante todo o ano (SILVA et al., 2014).

A vegetação da região é do tipo Caatinga, destacando-se as famílias Cactaceae e Bromeliaceae, das quais, se evidenciam as espécies Pilosocereus gounellei (F.A.C.Weber) Byles \& G.D. Rowley (xiquexique), Cereus jamacaru DC. (mandacaru) (LUCENA et al., 2013; SILVA et al., 2014). 
A comunidade possui uma escola estadual de ensino fundamental na comunidade Malhada Comprida, instalações comerciais, uma capela da igreja Católica localizada em Caruatá de Dentro, denominada de Capela de São Francisco, e dois salões da Associação de agricultores, também localizados em Caruatá de Dentro. Parte das crianças, e com mais frequência os adolescentes, estudam nas escolas da cidade e passam parte do dia por lá, já que têm acesso ao transporte concedido pelo município (LIMA et al., 2016).

Uma das principais economias locais é a agropecuária, em que se destaca a criação de caprinos e o cultivo de milho e feijão (IBGE, 2010), também, parte da renda da comunidade vem da aposentadoria dos mais velhos que em alguns casos são responsáveis pela educação dos netos, sedo algumas famílias separadas pela falta de opção de emprego e renda na região, deixando a cargo dos seus pais a educação dos seus filhos.

\section{Coleta e análise de dados}

As crianças e adolescentes pertemcem à comunidade São Francisco, zona rural de Cabaceiras, distante $13 \mathrm{~km}$ da sede do município, com aproximadamente 70 residências. Esse povoado está dividido em localidades denominadas de Caruatá de Dentro, Jerimum, Alto Fechado, Rio Direito e Malhada Comprida (LIMA et al., 2016).

Os dados foram coletados através de questionários aplicados em visitas às residências. As entrevistas foram realizadas com todas as crianças e adolescentes em dois momentos, com intervalo de dois anos, com objetivo de analisar os dados durante esse intervalo de tempo.

A primeira parte do trabalho de campo foi realizada no período de maio a setembro de 2011, durante os finais de semana onde os pesquisados ficavam alojados na sede de um grupo escolar desativado, cedido gentilmente pelos moradores da localidade do Rio Direito, possibilitando, por ser uma área central, para se visitar todas as casas da comunidade São Francisco.

Foram identificadas um total de 51 crianças e adolescente com faixa etária de seis a 15 anos. Do total de informantes identificados, 50 participaram do estudo e um se recusou a participar.

Foram coletados dados de 25 meninos ( 13 com idades de seis a 10 anos; e 12 acima de 10 anos) e 25 meninas (oito de seis a 10 anos, e 17 acima de 10 anos), todos em idade escolar, cursando da Alfabetização ao $2^{\circ}$ Ano do Ensino Médio.

A segunda parte da pesquisa foi realizada em outubro de 2013 , dois anos depois do primeiro contato com as crianças e adolescentes entrevistados. As crianças que anteriormente tinham seis anos, nessa ocasião estavam com oito, e os adolescentes antes com 15 anos, agora estavam com 17. Neste período, foram coletados dados de 33 crianças e adolescentes, distribuídos em 17 meninos (10 dos oito aos 14 anos, e sete dos 15 aos 17 anos) e 16 meninas (11 dos oito aos 13 anos, e cinco dos 14 aos 17 anos), cursando do $3^{\circ}$ Ano fundamental ao $3^{\circ}$ Ano do Ensino Médio. 
Nessa segunda ocasião, o número de jovens entrevistados foi menor do que na primeira parte do trabalho, pois parte dos entrevistados deixaram a comunidade com suas famílias.

Para as crianças e adolescentes foi explicado o objetivo do estudo e os pais, que concordaram em permitir a pesquisa com seus filhos, assinaram o Termo de Consentimento Livre e Esclarecido. Esta pesquisa foi aprovada pelo Comitê de Ética em Pesquisa do Hospital Lauro Wanderley da Universidade Federal da Paraíba (CEP/HULW no 297/11, Folha de Rosto no 420134).

A metodologia utilizada para pesquisa foi à entrevista semi-estruturada e lista livre de Quinlan (2005). Esta metodologia possui três características básicas que devem ser consideradas: 1 . As pessoas que são entrevistadas tendem a listar os termos por ordem de familiaridade; 2. Pessoas que sabem mais sobre um domínio tendem a citar mais que pessoas que sabem menos; 3 . Termos mais citados indicam itens mais proeminentes ou salientes localmente, sendo listados nomes de plantas sem distinção de origem (nativa e exótica).

No processo da entrevista as crianças/adolescentes, caracterizavam cada espécie citada de acordo com a sua relação e informação sobre o uso e conhecimento de cada planta mencionada, em várias categorias, relacionadas com: Alimentação, forragem, medicinal, sombra, combustível, construção, tecnologia, higiene e outros.

Os informantes foram estimulados, de acordo com a técnica "Reading back" (ler de volta: leia o que você escreveu ou releitura), quando o informante declarava não se lembrar de outros itens, o pesquisador lia lentamente todos os itens citados pelo informante, o que permitia aos mesmos adicionar itens não listados anteriormente .Observação participante e turnê guiada foram utilizados como métodos auxiliares para a confirmação dos dados (ALBUQUERQUE et al., 2010).

As plantas citadas pelos informantes foram coletadas, herborizadas, identificadas e tombadas na coleção do Herbário Jaime Coelho de Moraes (EAN), no Centro de Ciências Agrárias da Universidade Federal da Paraíba.

\section{Resultados}

Foram registradas, na primeira etapa da pesquisa, 192 plantas (Tabela 1), sendo identificadas 154 espécies, distribuídas em 46 famílias, das quais 53,73\% são exóticas e 46,27\% nativas. Obtiveram-se 1.902 citações, sendo $54 \%$ das citações de meninos e $46 \%$ de meninas

. No entanto, na segunda parte da pesquisa, foram registradas, 162 plantas (tabela 1), sendo identificadas 131 espécies, distribuídas em 52 famílias, das quais $56 \%$ são exóticas e $44 \%$ nativas, das quais foram obtidas 1.762 citações, sendo $54 \%$ das citações de meninos e $46 \%$ de meninas. 
Tabela 1: Lista de espécies botânicas citadas pelos entrevistados durante o estudo etnobotânico na comunidade São Francisco, Cabaceiras, Paraíba (Nordeste do Brasil).

\begin{tabular}{|c|c|c|c|}
\hline Família & Nome científico & $\begin{array}{l}\text { Nome } \\
\text { popular }\end{array}$ & Usos \\
\hline Agavaceae & Agave angustifolia Haw. & Agave & Ornamental \\
\hline Alliaceae & Allium cepa L. & Cebola & Alimentar \\
\hline Alliaceae & Allium cepa L. & Cebola roxa & Alimentar \\
\hline Anacardiaceae & Myracrodruon urundeuva Allemão & Aroeira & $\begin{array}{c}\text { Medicinal/ tecnologia/sombra/ } \\
\text { combustível/forragem/ construção }\end{array}$ \\
\hline Anacardiaceae & Schinus Terebinthifolius Raddi & $\begin{array}{l}\text { Aroeira da } \\
\quad \text { Praia }\end{array}$ & Forragem / combustível \\
\hline Anacardiaceae & Schinopsis brasiliensis Engl. & Baraúna & $\begin{array}{l}\text { Sombra/tecnologia/construção/ } \\
\text { combustível forragem }\end{array}$ \\
\hline Anacardiaceae & Anacardium occidentale L. & Caju & Alimentar \\
\hline Anacardiaceae & Mangifera indica L. & Manga & Alimentar \\
\hline Anacardiaceae & Spondias purpurea L. & Siriguela & Alimentar \\
\hline Anacardiaceae & Spondias sp. & Umbu cajá & Alimentar \\
\hline Anacardiaceae & Spondias tuberosa L. & Umbuzeiro & Alimentar \\
\hline Annonaceae & Annona muricata $\mathrm{L}$. & Graviola & Alimentar \\
\hline Annonaceae & Annona squamosa $\mathrm{L}$. & Maçã & Alimentar \\
\hline Annonaceae & Annona squamosa L. & Pinha & Alimentar \\
\hline Apiaceae & Daucus carota L. & Cenoura & Alimentar \\
\hline Apiaceae & Coriandrum sativum $\mathrm{L}$. & Coentro & Alimentar \\
\hline Apiaceae & Anethum graveolens L. & Endro & Medicinal \\
\hline Apiaceae & Foeniculum vulgare Mill. & Erva doce & Medicinal \\
\hline Apocynaceae & Catharanthus roseus (L.) G. Don. & $\begin{array}{l}\text { Boa noite / } \\
\text { bom dia }\end{array}$ & Ornamental \\
\hline Apocynaceae & Plumeria pudica Jacq. & $\begin{array}{l}\text { Buquê de } \\
\text { Noiva }\end{array}$ & Ornamental \\
\hline Apocynaceae & Hancornia speciosa Gomes & Mangaba & Alimentar \\
\hline Apocynaceae & Aspidosperma pyrifolium Mart. & Pereiro & $\begin{array}{c}\text { Sombra/forragem/construção/com } \\
\text { bustível }\end{array}$ \\
\hline Araceae & Deiffembachia amoena Schott & $\begin{array}{l}\text { Comigo } \\
\text { ninguém pode }\end{array}$ & Ornamental \\
\hline Arecaceae & Euterpe oleracea Mart. & Açaí & Alimentar \\
\hline Arecaceae & $\operatorname{Cocos} n$ & Coqueiro & $\begin{array}{l}\text { Alimentar/construção/tecnologia/ } \\
\text { sombra }\end{array}$ \\
\hline $\begin{array}{l}\text { Arecaceae } \\
\text { Asteraceae }\end{array}$ & $\begin{array}{l}\text { Indeterminado } \\
\text { Lactuca sp. }\end{array}$ & $\begin{array}{l}\text { Palmeira } \\
\text { Alface }\end{array}$ & $\begin{array}{l}\text { Ornamental } \\
\text { Alimentar }\end{array}$ \\
\hline Asteraceae & Camomilla recutita (L.) Rauschert & Camomila & Medicina \\
\hline Asteraceae & Helianthus annuus L. & Girassol & Ornamental \\
\hline Asteraceae & Indeterminado & Margarida & Ornamental \\
\hline Bignoniaceae & $\begin{array}{c}\text { Tabebuia aurea (Silva } \\
\text { Manso)Benth. Hook. F.ex. S. Moore }\end{array}$ & Cardeiro & $\begin{array}{c}\text { Forragem/construção/ } \\
\text { combustível }\end{array}$ \\
\hline Big & $\begin{array}{c}\text { Tabebuia aurea (Silva } \\
\text { Manso)Benth. Hook. F.ex. S. Moore }\end{array}$ & Craibeira & $\begin{array}{l}\text { Sombra/construção/tecnologia/ } \\
\text { forragem }\end{array}$ \\
\hline Bignoniaceae & Tabebuia sp & Ipê & Ornamental/ sombra \\
\hline
\end{tabular}

Continua... 
...continuação.

\begin{tabular}{|c|c|c|c|c|}
\hline Família & Nome científico & $\begin{array}{c}\text { Nome } \\
\text { popular }\end{array}$ & \multicolumn{2}{|l|}{ Usos } \\
\hline Bignoniaceae & Tabebuia sp & Ipê roxo & \multicolumn{2}{|l|}{ Ornamental } \\
\hline Bixaceae & Bixa orellana $\mathrm{L}$. & Urucum & \multicolumn{2}{|l|}{ Alimentar } \\
\hline Brassicaceae & Brassica oleracea L. & Couve & \multicolumn{2}{|l|}{ Alimentar } \\
\hline Brassicaceae & Brassica oleracea var. botrytis & Couve flor & \multicolumn{2}{|l|}{ Alimentar } \\
\hline Brassicaceae & Brassica sylvestris (L.) Mill. & Repolho & \multicolumn{2}{|l|}{ Alimentar } \\
\hline Bromeliaceae & Ananas comosus (L.) Merr. & Abacaxi & \multicolumn{2}{|l|}{ Alimentar } \\
\hline Bromeliaceae & Bromelia laciniosa Mart. ex Schult. f. & Macambira & \multicolumn{2}{|l|}{ Forragem } \\
\hline Burseraceae & $\begin{array}{c}\text { Commiphora leptaphbeos (Mart.) } \\
\text { J.B.Gillet }\end{array}$ & Imburana & \multicolumn{2}{|l|}{$\begin{array}{c}\text { Sombra/construção/forragem/com } \\
\text { bustível }\end{array}$} \\
\hline Cactaceae & $\begin{array}{c}\text { Melocactus bahiensis (Britton \& } \\
\text { Rose) Luetzelb. }\end{array}$ & $\begin{array}{l}\text { Coroa de } \\
\text { Frade }\end{array}$ & \multicolumn{2}{|l|}{ Ornamental/alimentação/forragem } \\
\hline Cactaceae & $\begin{array}{c}\text { Tacinga inamoena N.P.Taylor e } \\
\text { Stuppy }\end{array}$ & Cumbeba & \multicolumn{2}{|l|}{ Ornamental } \\
\hline Cactaceae & Pilocereus pachyclodus (F.) Ritter & Facheiro & \multicolumn{2}{|l|}{$\begin{array}{l}\text { Alimentar/forragem/construçãc } \\
\text { combustível }\end{array}$} \\
\hline Cactaceae & Cereus jamacaru DC. & Mandacaru & \multirow{2}{*}{\multicolumn{2}{|c|}{$\begin{array}{l}\text { Ornamental /alimentar } \\
\text { /forragem/higiene - pessoal }\end{array}$}} \\
\hline Cactaceae & Opuntia ficus-indica (L.) Mill & Palma & & \\
\hline Cactaceae & $\begin{array}{c}\text { Tacinga palmadora N.P.Taylor e } \\
\text { Stuppy }\end{array}$ & Palmatória & \multicolumn{2}{|l|}{ Forragem } \\
\hline Cactaceae & $\begin{array}{c}\text { Pilocereus gounellei (F.A.C. Weber) } \\
\text { Byles \& Rowley }\end{array}$ & Xique-xique & \multicolumn{2}{|l|}{ Forragem /alimentar } \\
\hline Caesalpiniaceae & Tamarindus indica $\mathrm{L}$. & Tamarindo & \multicolumn{2}{|l|}{ Alimentar } \\
\hline Caprifoliaceae & $\begin{array}{c}\text { Sambucus australis Cham. \& } \\
\text { Schltdl. O }\end{array}$ & Sabugueiro & \multicolumn{2}{|l|}{ Indeterminado } \\
\hline Caricaceae & Carica papaya L. & Mamão & \multicolumn{2}{|l|}{ Alimentar } \\
\hline Chenopodiaceae & Beta vulgaris L. var. esculenta & Beterraba & \multicolumn{2}{|l|}{ Alimentar } \\
\hline Chenopodiaceae & Chenopodium ambrosioides L. & Mastruz & \multicolumn{2}{|l|}{ Medicinal } \\
\hline Combretaceae & Terminalia catappa Linn & Castanhola & \multicolumn{2}{|l|}{ Sombra/medicinal/alimentação } \\
\hline $\begin{array}{l}\text { Combretaceae } \\
\text { Convolvulaceae }\end{array}$ & $\begin{array}{l}\text { Thiloa glaucocarpa (Mart.) Eichler } \\
\text { Ipomoea batatas (L.) Lam. }\end{array}$ & $\begin{array}{l}\text { João Mole } \\
\text { Batata doce }\end{array}$ & \multicolumn{2}{|l|}{$\begin{array}{l}\text { Medicinal } \\
\text { Alimentar }\end{array}$} \\
\hline Crassulaceae & Sedum morganianum Walth & $\begin{array}{l}\text { Dedinho de } \\
\text { anjo }\end{array}$ & \multicolumn{2}{|l|}{ Ornamental } \\
\hline Crassulaceae & Bryophyllum calycinum Salisb & Saião & \multicolumn{2}{|l|}{ Medicinal } \\
\hline Cucurbitaceae & Cucurbita sp. & Jerimum & \multicolumn{2}{|l|}{ Alimentar } \\
\hline Cucurbitaceae & Cucumis anguria $\mathrm{L}$. & Maxixe & \multicolumn{2}{|l|}{ Alimentar } \\
\hline Cucurbitaceae & $\begin{array}{l}\text { Citrullus lanatus (Thunb.) Matsum. } \\
\text { \& Nakai }\end{array}$ & Melancia & \multicolumn{2}{|l|}{ Alimentar } \\
\hline Cucurbitaceae & Cucumis melo L. & Melão & \multicolumn{2}{|l|}{ Alimentar } \\
\hline Cucurbitaceae & Cucumis sativus $\mathrm{L}$. & Pepino & \multicolumn{2}{|l|}{ Alimentar } \\
\hline Curcubitaceae & Cucurbita pepo L. & Abóbora & \multicolumn{2}{|l|}{ Alimentar } \\
\hline Curcubitaceae & Sechium edule (Jacq.) Sw. & Chuchu & Alimentar & \\
\hline Euphorbiaceae & Euphorbia tirucalli Linn & Aveloz & Veneno/combustível & \\
\hline & & Revbea, São P & aulo, V. 13, Nº 4: 390-407, 2018. & $\begin{array}{l}\begin{array}{c}\text { revista brasileira } \\
\text { de } \\
\text { educação } \\
\text { ambiental } \\
397\end{array}\end{array}$ \\
\hline
\end{tabular}


...continuação.

\begin{tabular}{|c|c|c|c|}
\hline Família & Nome científico & $\begin{array}{c}\text { Nome } \\
\text { popular }\end{array}$ & Usos \\
\hline $\begin{array}{l}\text { Euphorbiaceae } \\
\text { Euphorbiaceae }\end{array}$ & $\begin{array}{l}\text { Cnidoscolus quercifolius Pohl. } \\
\text { Phyllantus acidus (L.) Skeels }\end{array}$ & $\begin{array}{l}\text { Favela } \\
\text { Groselha }\end{array}$ & $\begin{array}{l}\text { Medicinal } \\
\text { Alimentar }\end{array}$ \\
\hline Euphorbiaceae & Croton blanchetianus Baill. & Marmeleiro & $\begin{array}{l}\text { Forragem/medicinal/veneno/ } \\
\text { construcão }\end{array}$ \\
\hline Euphorbiaceae & Jatropha mollissima (Pohl) Baill. & Pinhão & Medicinal \\
\hline Euphorbiaceae & Jatropha curcas L. & $\begin{array}{l}\text { Pinhão } \\
\text { branco }\end{array}$ & Medicinal \\
\hline Euphorbiaceae & Jatropha gossupiifolia L. & Pinhão roxo & Medicinal \\
\hline Euphorbiaceae & $\begin{array}{c}\text { Cnidoscolus loefgrenii (Pax \& K. } \\
\text { Hoffm.) Pax \& K. Hoffm. }\end{array}$ & Urtiga & Veneno \\
\hline Fabaceae & Arachis hypogaea $\mathrm{L}$. & Amendoim & Alimentar \\
\hline Fabaceae & Prosopis juliflora (Sw.) DC. & Algaroba & $\begin{array}{l}\text { Forragem/ construção/ sombra/ } \\
\text { combustível/ tecnologia }\end{array}$ \\
\hline Fabaceae & Anadanthera colubrina (Vell.) & Angico & $\begin{array}{c}\text { Medicinal/ sombra/ forragem / } \\
\text { tecnologia }\end{array}$ \\
\hline Fabaceae & Poincianella pyramidalis Tul. & Catingueira & $\begin{array}{l}\text { Combustível/ construção/sombra/ } \\
\text { medicinal }\end{array}$ \\
\hline Fabaceae & Phaseolus vulgaris $\mathrm{L}$. & Feijão & Alimentar \\
\hline Fabaceae & $\begin{array}{c}\text { Delonix regia (Bojer ex Hook.) } \\
\text { Raf. }\end{array}$ & Flamboiã & Ornamental/sombra \\
\hline Fabaceae & Hymenoca courbaril L. & Jatobá & Medicinal \\
\hline Fabaceae & $\begin{array}{c}\text { Libidibia ferrea (Mart. ex Tul.) } \\
\text { L.P. Queiroz }\end{array}$ & Jucá & $\begin{array}{c}\text { Tecnologia/forragem/combustível/ } \\
\text { sombra }\end{array}$ \\
\hline Fabaceae & Mimosa teniuflora (Willd) Poir & Jurema & $\begin{array}{c}\text { Forragem/combustível/construção/ } \\
\text { medicinal/ tecnologia/sombra }\end{array}$ \\
\hline Fabaceae & $\begin{array}{c}\text { Piptadenia stipulaceae (Benth.) } \\
\text { Ducke }\end{array}$ & $\begin{array}{l}\text { Jurema } \\
\text { Branca }\end{array}$ & Combustível/forragem \\
\hline Fabaceae & $\begin{array}{c}\text { Leucaena leucocephala (Lam.) } \\
\text { de Wit }\end{array}$ & Leucena & Forragem/ sombra/tecnologia \\
\hline Fabaceae & Erythrina velutina Willd. & Mulungú & $\begin{array}{c}\text { Ornamental/ sombra/ forragem / } \\
\text { veneno/ tecnologia }\end{array}$ \\
\hline Fabaceae & $\begin{array}{l}\text { Bauhinia cheilantha (Bong.) } \\
\text { Steud. }\end{array}$ & Pata de vaca & Medicinal \\
\hline $\begin{array}{l}\text { Fabaceae } \\
\text { Fabaceae } \\
\text { Lamiaceae }\end{array}$ & $\begin{array}{l}\text { Caesalpinia echinata Lam. } \\
\text { Clitoria fairchildiana Howard } \\
\text { Plectrantrus barbatus Andrews }\end{array}$ & $\begin{array}{l}\text { Pau-brasil } \\
\text { Sombreiro } \\
\text { Boldo }\end{array}$ & $\begin{array}{l}\text { Construção } \\
\text { Sombra } \\
\text { Medicinal }\end{array}$ \\
\hline Lamiaceae & $\begin{array}{c}\text { Plectranthus amboinicus (Lour.) } \\
\text { Spreng }\end{array}$ & $\begin{array}{l}\text { Hortelã } \\
\text { Graúda }\end{array}$ & Medicinal \\
\hline Lamiaceae & Mentha arvensis $\mathrm{L}$. & $\begin{array}{l}\text { Hortelã } \\
\text { Miúda }\end{array}$ & Medicinal \\
\hline Lauraceae & Persea americana Mill. & Abacate & Medicinal / alimentar \\
\hline Lauraceae & Laurus nobilis L. & Louro & Medicinal \\
\hline $\begin{array}{l}\text { Liliaceae } \\
\text { Liliaceae }\end{array}$ & $\begin{array}{c}\text { Allium sativum } \mathrm{L} \text {. } \\
\text { Aloe vera }(\mathrm{L} \text {.) Burm.f. }\end{array}$ & $\begin{array}{l}\text { Alho } \\
\text { Babosa }\end{array}$ & $\begin{array}{l}\text { Alimentar } \\
\text { Medicinal }\end{array}$ \\
\hline Liliaceae & Sansevieria trifasciata Hort & $\begin{array}{l}\text { Espada de } \\
\text { São Jorge }\end{array}$ & Ornamental \\
\hline Losaceae & $\begin{array}{c}\text { Aosa rupestris(Gardner) } \\
\text { Weigend }\end{array}$ & Urtiga branca & Veneno \\
\hline Malpighiaceae & Malphighia glabra L. & Acerola & Alimentar \\
\hline Malvaceae & Gossypium sp. & Algodão & Higiene pessoal \\
\hline
\end{tabular}


...continuação.

\begin{tabular}{|c|c|c|c|c|}
\hline Família & Nome científico & $\begin{array}{l}\text { Nome } \\
\text { popular }\end{array}$ & Usos & \\
\hline Malvaceae & Hibiscus rosa-sinensis L. & Beijo & Ornamental & \\
\hline Malvaceae & Melochia corchorifolia L. & Capa bode & Forragem/ tecnologia & \\
\hline Malvaceae & Hibiscus sinensis Mill. & $\begin{array}{l}\text { Estrela da } \\
\text { noite }\end{array}$ & Ornamental & \\
\hline Meliaceae & Azadirachta indica A. Juss. & $\mathrm{Nim}$ & $\begin{array}{l}\text { Veneno/ sombra/ combustível/ } \\
\text { construção/ forragem }\end{array}$ & \\
\hline Moraceae & Morus sp. & Amora & Alimentar & \\
\hline Moraceae & Ficus benjamina L. & Fícus & Sombra/ornamental/ forragem & \\
\hline Moraceae & Artocarpus integrifolia Lf. & Jaca & Alimentar & \\
\hline Musaceae & Musa sp. & Banana & Alimentar & \\
\hline Myrtaceae & $\begin{array}{l}\text { Syzygium jacubolanum } \\
\text { (Lam.) DC }\end{array}$ & Azeitona & Alimenta/sombea/forragem & \\
\hline $\begin{array}{l}\text { Myrtaceae } \\
\text { Myrtaceae }\end{array}$ & $\begin{array}{c}\text { Psidium guajava L. } \\
\text { Myrcyaria cauliflora Berg. }\end{array}$ & $\begin{array}{l}\text { Goiaba } \\
\text { Jabuticaba }\end{array}$ & $\begin{array}{l}\text { Alimentar } \\
\text { Alimentar }\end{array}$ & \\
\hline Myrtaceae & $\begin{array}{c}\text { Syzygium malaccense (L.) } \\
\text { Merr \& Perry }\end{array}$ & Jambo & Alimenta & \\
\hline Myrtaceae & Eugenia uniflora L. & Pitanga & Alimentar & \\
\hline Nyctaginaceae & Bougainvillea glabra Choisy & Primavera & Ornamental & \\
\hline Nyctaginaceae & $\begin{array}{l}\text { Bougainvillea spectabilis } \\
\text { Willd }\end{array}$ & $\begin{array}{l}\text { Rainha do } \\
\text { Prado }\end{array}$ & Ornamental & \\
\hline Oxalidaceae & Averrhoa carambola L. & Carambola & Alimentar & \\
\hline Passifloreaceae & Passiflora edulis Sims. & Maracujá & Alimentar & \\
\hline $\begin{array}{l}\text { Poaceae } \\
\text { Poaceae }\end{array}$ & $\begin{array}{l}\text { Oryza sativa L. } \\
\text { Saccharum } \mathrm{sp} .\end{array}$ & $\begin{array}{l}\text { Arroz } \\
\text { Cana }\end{array}$ & $\begin{array}{l}\text { Alimentar } \\
\text { Alimentar }\end{array}$ & \\
\hline Poaceae & $\begin{array}{l}\text { Cymbopogon citratus (DC) } \\
\text { Stapf. }\end{array}$ & $\begin{array}{l}\text { Capim } \\
\text { Cidreira }\end{array}$ & Medicinal & \\
\hline $\begin{array}{l}\text { Poaceae } \\
\text { Poaceae }\end{array}$ & $\begin{array}{l}\text { Indeterminado } \\
\text { Cenchrus ciliaris }\end{array}$ & $\begin{array}{l}\text { Capim } \\
\text { Capim Búfalo }\end{array}$ & $\begin{array}{l}\text { Forragem } \\
\text { Forragem }\end{array}$ & \\
\hline Poaceae & $\begin{array}{l}\text { Pennisetum purpureum } \\
\text { Schumach }\end{array}$ & $\begin{array}{l}\text { Capim } \\
\text { Elefante }\end{array}$ & Forragem & \\
\hline Poaceae & Zea mays L. & Milho & Alimentar & \\
\hline Poaceae & Sorghum vulgare Pers. & Sorgo & Sombra & \\
\hline Punicaceae & Punica granatum L. & Romã & $\begin{array}{l}\text { Alimentar /medicinal } \\
\text { Sombra/alimentacão/combustíve }\end{array}$ & \\
\hline Rhamnaceae & Ziziphus joazeiro Mart. & Juazeiro & $\begin{array}{c}\text { higiene pessoal } \\
\text { /forragem/medicinal/construção }\end{array}$ & \\
\hline Rhamnaceae & $\begin{array}{c}\text { Talisia esculenta (A. St. Hil.) } \\
\text { Roldk. }\end{array}$ & Pitomba & Alimental & \\
\hline Rosaceae & Prunus sp. & Cerejeira & Alimentar & \\
\hline Rosaceae & Fragaria vesca L. & Morango & Alimentar & \\
\hline Rosaceae & Pyrus communis $\mathrm{L}$. & Pêra & Alimentar & \\
\hline Rosaceae & Prunus persica (L.) Batsch & Pêssego & Alimentar & \\
\hline Rosaceae & Rosa sp. & Rosa & Ornamental & \\
\hline \multirow[t]{2}{*}{ Rutaceae } & Ruta graveolens L. & Arruda & Medicinal/ místico /religioso & \\
\hline & & \multicolumn{2}{|c|}{ Revbea, São Paulo, V. 13, № 4: 390-407, 2018.} & 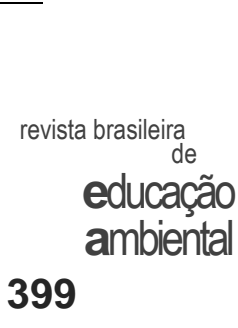 \\
\hline
\end{tabular}


...continuação.

\begin{tabular}{|c|c|c|c|}
\hline Família & Nome científico & $\begin{array}{l}\text { Nome } \\
\text { popular }\end{array}$ & Usos \\
\hline Rutaceae & Ruta graveolens L. & Arruda & Mágico- religioso/medicinal \\
\hline Rutaceae & Coffea arabica $\mathrm{L}$ & Café & Alimentar \\
\hline Rutaceae & Citrus aurantium L. & Laranja & Alimentar \\
\hline Rutaceae & Citrus limon L. Burmann f. & Limão & Alimentar \\
\hline Sapotaceae & $\begin{array}{l}\text { Sideroxylon obtusifolium } \\
\text { (Roem \& Schutt) T. D. Penn }\end{array}$ & Quixabeira & Alimentação /forragem /sombra \\
\hline Scrophulariaceae & Scoparia dulcis L. & Vassourinha & Tecnologia \\
\hline Solanaceae & Solanum tuberosum $\mathrm{L}$. & Batata & Alimentar \\
\hline Solanaceae & Nocotiana glauca Graham & Oliveira & Alimenta/construção/forragem \\
\hline Solanaceae & Capsicum annuum L. & Pimentão & Alimentar \\
\hline Solanaceae & Capsicum sp. & Pimenta & Alimentar \\
\hline Solanaceae & $\begin{array}{c}\text { Lycopersicon esculentum } \\
\text { Mill. }\end{array}$ & Tomate & Alimentar \\
\hline Theaceae & $\begin{array}{c}\text { Camellia sinensis (L.) } \\
\text { Kuntze }\end{array}$ & Chá preto & Alimentar \\
\hline Verbenaceae & Lippia alba (Mill) N.E.Br & Erva cidreira & Medicinal \\
\hline Vitaceae & Vitis sp. & Uva & Alimentar \\
\hline Zingiberaceae & Zingiber officinale Roscoe & Gengibre & Medicinal \\
\hline Indeterminado & Indeterminado & $\begin{array}{l}\text { Algodão do } \\
\text { Pará }\end{array}$ & Higiene pessoal/ sombra \\
\hline Indeterminado & Indeterminado & Brasileirinho & Ornamental \\
\hline Indeterminado & Indeterminado & Cabaça & Artesanal \\
\hline Indeterminado & Indeterminado & Cacto & Ornamental \\
\hline Indeterminado & Indeterminado & $\begin{array}{l}\text { Cacho de } \\
\text { noiva }\end{array}$ & Ornamental \\
\hline Indeterminado & Indeterminado & Camapú & Forragem/medicinal \\
\hline Indeterminado & Indeterminado & Carrapicho & Outro \\
\hline Indeterminado & Indeterminado & Cominho & Alimentar \\
\hline Indeterminado & Indeterminado & Fava & Alimentar \\
\hline Indeterminado & Indeterminado & Gerânio & Ornamental \\
\hline Indeterminado & Indeterminado & Gramas & Ornamental \\
\hline Indeterminado & Indeterminado & Graveto & Forragem/combustível \\
\hline Indeterminado & Indeterminado & Imbé & Ornamental \\
\hline Indeterminado & Indeterminado & Imbiratã & Medicinal \\
\hline Indeterminado & Indeterminado & Macela & Medicinal \\
\hline Indeterminado & Indeterminado & Onze horas & Ornamenta \\
\hline Indeterminado & Indeterminado & $\begin{array}{l}\text { Quebra } \\
\text { Pedra }\end{array}$ & Medicinal \\
\hline Indeterminado & Indeterminado & Relva & Ornamental \\
\hline Indeterminado & Indeterminado & Sete dores & Medicinal \\
\hline Indeterminado & Indeterminado & Ubiratã & Medicinal \\
\hline
\end{tabular}


As plantas identificadas nesta primeira parte foram classificadas dentro das categorias de uso alimentação, forragem, medicinal, sombra, combustível, construção, tecnologia, higiene e outros (Figura 2).

Nas categorias de uso, tanto na primeira parte da pesquisa quanto na segunda, a alimentação, forragem, sombra, combustível e construção foram as categorias que receberam maior número de citações. A categoria sombra se destacou pelo crescimento das citações na segunda parte da pesquisa, que também foi refletido nas declarações dos informantes:

"O Nim é uma planta importante porque faz sombra e ajuda a gente a respirar" R.D, 12anos.

"A castanhola serve pra fazer sombra, se não fosse as árvores, a gente não tava vivendo, porque as arvores que trazem o ar" M.C , 9 anos.

A categoria alimentação obteve o maior número de citações e a espécie que mais se destacou, para os entrevistados da primeira parte da pesquisa, foi Opuntia ficus indica (L.) (Mill) (Palma) com 7\% (47) das observações, em que 30 apresentaram o cladódio como parte mais citada, seguida do fruto com 17 citações. Ainda na categoria alimentação, o umbuzeiro Spondias tuberosa, com 39 citações $(5,81 \%)$ foi a segunda planta mais citada e teve como parte mais referida o fruto com $36(92 \%)$.

Assim como a primeira parte da pesquisa, a categoria alimentação (Figura 2) se manteve como a categoria mais citada na segunda parte da pesquisa, e a espécie que mais se destacou para os entrevistados foi Spondias tuberosa (umbuzeiro) com 34 observações $(7,09 \%)$ das quais 33 (99\%) apresentaram o fruto como parte mais citada, seguida da acerola (Malpighia glabra L.) que obteve o segundo maior número de citações, com 25 citações $(5,22 \%)$, obtendo o fruto a totalidade de parte mais citada.
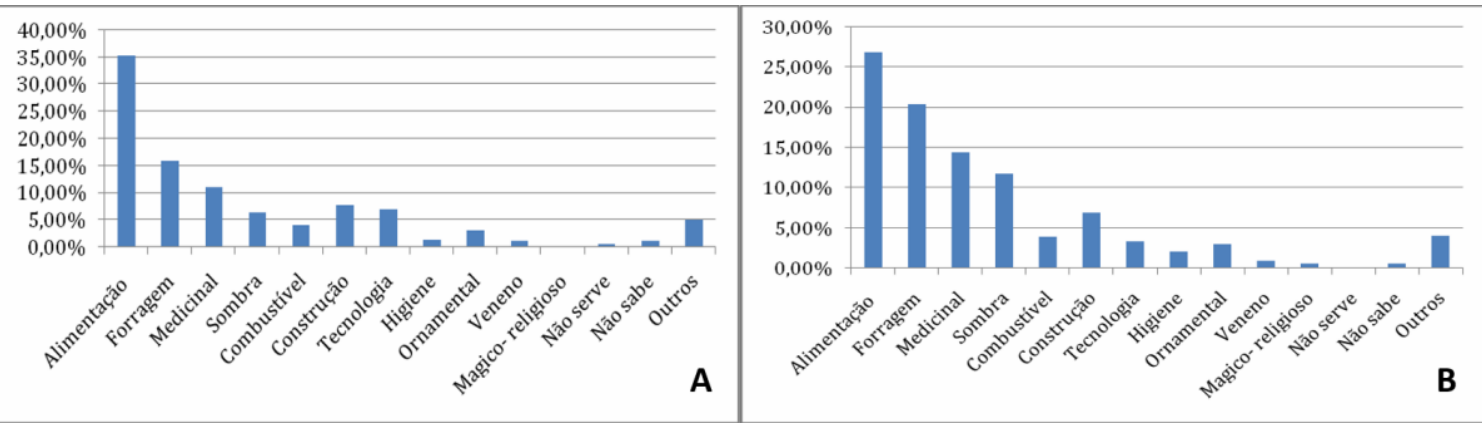

Figura 2: Percentual da forma de utilização das plantas citadas por categorias na comunidade São Francisco, Cabaceiras, Paraíba (Nordeste do Brasil). O gráfico A corresponde a primeira etapa da pesquisa e $\mathbf{B}$ a segunda etapa. 
A faixa etária que apresentou um número maior de citações, na primeira parte da pesquisa (Figura 3), foi a de 10 aos 12 anos com $44 \%$ das citações. Já as crianças dos seis aos nove anos obtiveram $31 \%$ das citações, e os adolescentes 13 aos 15 anos, com a menor porcentagem citada, perfazendo $25 \%$. No segundo momento da pesquisa (Figura 3), a faixa etária que se destacou com os maiores resultados foi a dos 12 aos 14 anos perfazendo um total de $56,31 \%$, seguidos das crianças de oito aos 12 anos com $31,61 \%$ e por último os adolescentes com de 15 a 17 anos com um percentual de 20,37\%.

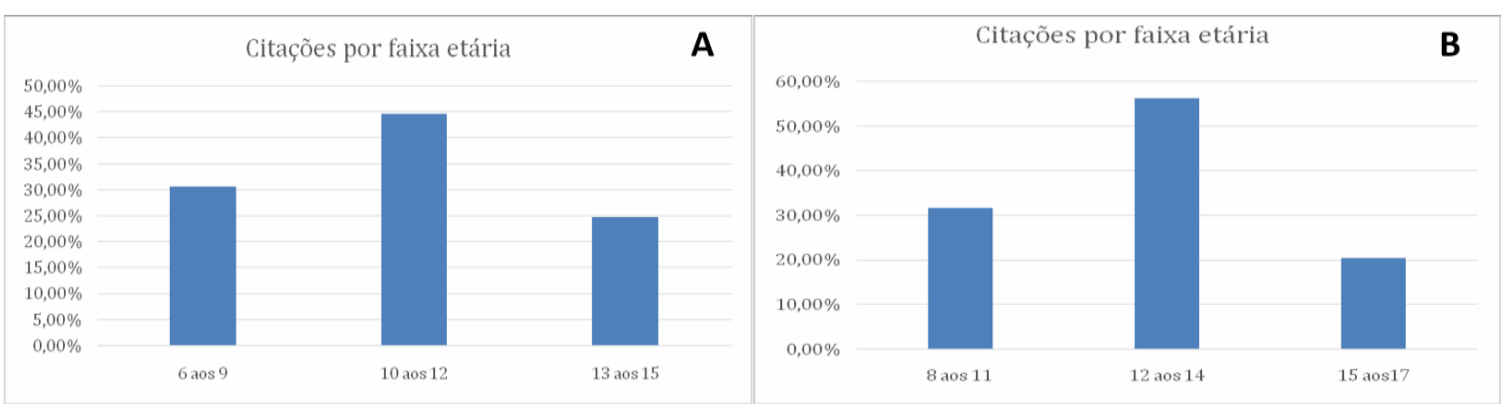

Figura 3: Percentual de citações por faixa etária na comunidade São Francisco, Cabaceira, Paraíba (Nordeste do Brasil). Onde o gráfico A corresponde a primeira etapa da pesquisa e B a segunda etapa.

Como forragem, na primeira parte da pesquisa, O. fícus indica (L.) Mill, também ocupou lugar de destaque entre as citações, sendo citada 39 vezes, obtendo o cladódio como parte utilizada (37 citações), já Prosopis juliflora (Algaroba) foi a segunda espécie mais citada da categoria, recebendo, 29 citações (96\%), das quais a vagem (fruto), quase em sua totalidade, 28 vezes (99\%), foi citada como parte mais utilizada na alimentação animal.

A segunda parte da pesquisa não seguiu a mesma linha da primeira quando a categoria citada foi a forragem, demonstrando como destaque nas citações $P$. juliflora, com 40 citações $(11,33 \%)$, sendo a vagem citada 28 vezes $(70 \%)$ e as folhas 12 vezes, seguida da algaroba, O. ficus indica (L.) Mill, obteve 28 citações, citando o cladódio como parte mais utilizada.

Para categoria medicinal na primeira parte da pesquisa Jatropha gossypiifolia L. (Pinhão) apresentou 37 citações (17,62\%), com 54,05\% como indicações para ferimentos (cicatrização), obtendo o látex $100 \%$ das citações. Outra planta que também se destacou na categoria foi o boldo com16 citações $(7,62 \%)$, com indicações para dores de barriga sendo as folhas $100 \%$ das partes citadas.

Ainda na categoria medicinal, na segunda parte da pesquisa, o Jatropha gossypiifolia $L$ também obteve a maior parte das citações, 35 (13,62\%), como indicações para ferimentos (cicatrização), obtendo o látex $100 \%$ das citações; em seguida Mentha arvensis L. (hortelã miúda) com 24 citações $(9,34 \%)$ e indicação para problemas respiratórios. 
Para a categoria combustível, $P$. juliflora foi a mais citada com 22 citações $(28,20 \%)$, sendo utilizada $72 \%$ para fabricação de carvão, e $28 \%$ para lenha, seguida de Croton blanchetianus Baill (marmeleiro) com 08 citações $(10,26 \%)$, sendo utilizado $50 \%$ como lenha e $50 \%$ como carvão.

$\mathrm{Na}$ segunda parte da pesquisa, na categoria combustível, $P$. juliflora continuou sendo a espécie mais citada (28,57\%), tendo a utilização como lenha de $50 \%$ e como carvão de 50\%, seguida da Myracrodruon urundeuva Allemão (aroeira) com nove citações (12\%) tendo como utilização o carvão (56\%) e a lenha (44\%).

Semelhante às duas fases da pesquisa, tanto $P$. juliflora como Aspidosperma pyrifolium Mart (pereiro), obtiveram respectivamente $17(11,26 \%)$ e $26(21,31 \%)$ citações, seguidos de23 (15\%) e (23), (18,7\%) citações na categoria construção.

$\mathrm{Na}$ categoria sombra, Azadirachta indica A. Juss (nim) foi a planta mais citada, primeira pesquisa com12 citações $(9,84 \%)$, seguido do Umbuzeiro apresentando nove citações (7,38\%).

No entanto com referência a categoria sombra $S$. Tuberosa representou o primeiro lugar com 25 citações $(11,74 \%)$, seguido d a $P$. juliflora com 19 representações $(8,92 \%)$.

\section{Discussão}

O maior número de citações foi obtido com as crianças na faixa etária de 10 a 12 anos (44\%), resultado semelhante foi encontrado em estudos realizados na Tailândia (SETALAPHRUK, 2007) e no México (WYNDHAM, 2009), onde esses autores informaram que nessa faixa etária as crianças adquirem maior parte do seu conhecimento, e tendem a ter maior sensibilidade em relação ao meio ambiente e seus recursos naturais.

$\mathrm{Na}$ segunda parte da pesquisa, em que a faixa etária dos informantes foram dos adolescentes mais velhos, culminou em menor número de citações registradas, se assemelhando aos resultados encontrados na Índia (GARCíA, 2006), onde a autora relata que o contato com o meio ambiente e seus recursos naturais, diminuiu com a idade e com a falta de tempo, já que as crianças mais velhas se deslocam do campo para a escola na cidade, fato este também observado na comunidade São Francisco em que os informantes adolescentes obtiveram menor número de citações, uma vez que todos frequentavam as escolas da cidade de Cabaceiras, talvez assim distanciando-se da vivência rural e do contato com as plantas de seu meio e, provavelmente, diminuindo seu interesse pelas mesmas.

Dentre as plantas mais citadas, Opuntia ficus indica L. Mill (Palma) se destacou nas categorias alimentação e forragem. A grande importância para essa espécie foi encontrada em estudos realizados na região nordeste do Brasil por Lucena (2013) e Albuquerque (2005), onde esses autores citam a relação das comunidades estudadas com essa espécie exótica que é bem adaptada na região 
e utilizada frequentemente como alimentação animal. Os informantes declararam ainda ter obtido um amplo conhecimento para os diversos usos dessa espécie por meios de cursos oferecidos na comunidade.

Spondias tuberosa (umbuzeiro), espécie nativa, se destacou em vários momentos da pesquisa, sobretudo nas categorias alimentação e sombra, a espécie é citada por Lins Neto et al. (2010) e como "planta sagrada" para os nordestinos e expressada, como tal, em uma das declarações dadas por um dos informantes da pesquisa:

“...serve sim, mas ninguém vai desperdiçar o umbuzeiro pra fazer cerca” (R.D.F.S. 9 anos, a respeito do uso madeireiro de $S$. tuberosa)

Prosopis juliflora que também foi uma das espécies mais citadas na pesquisa, tanto na primeira, quanto na segunda fase, apareceu com destaque nas categorias forragem, combustível e sombra, Guerra et al. (2014) em sua pesquisa reporta o mesmo potencial utilitário dessa planta tratando dos usos madeireiros (combustível, construção, e tecnologia) e não-madeireiros (alimento, forrageiro, sombra, mágico-religioso) realizados por comunidades locais.

O fato de $P$. juliflora ter sido citada em várias categorias chamou a atenção na pesquisa, pois as plantas exóticas tiveram número de citação superior às nativas. Esse fato pode ser justificado uma vez que os informantes tendem a citar um número maior de espécies que fazem parte do seu cotidiano e vivem em seu entorno, segundo Nascimento et al. (2014), P. juliflora encontra-se bem estabelecida dentro do bioma Caatinga tendo sido introduzida em função de suas utilidades.

\section{Conclusão}

As diferentes informações que surgiram nas distintas etapas deste estudo reforçam a importância de realizar estudos etnobotânicos com crianças e jovens. Já que muitos destes citam plantas de origenm exótica, onde os mesmos mencionam nunca terem contato em campo com algumas destas espécies.

A quantidade de espécies nativas e exóticas conhecidas, mesmo por crianças que afirmam nunca ter tido contato com nenhum espécime demonstra o reflexo da influência familiar na construção da propriedade cultural e intelectual do indivíduo jovem.

Utilizar a etnobotânica como ferramenta de pesquisa pode levar à construção, junto a comunidades tradicionais, de formas específicas de manejo dos recursos naturais em cada ecossistema, aliando o conhecimento sobre plantas com a representatividade em grau de importância, propondo a elaboração de estratégias que garantam a conservação da diversidade local, buscando autores do cotidiano, que em seu crescimento, se nutram também de uma cultura Revbea, São Paulo, V. 13, № 4: 390-407, 2018. 
repassada de gerações. Essas ações podem ser realizadas em pesquisas futuras e com ferramentas de educação ambiental, levando essas crianças e adolescentes a terem uma visão mais acurada sobre a importância dos recursos naturais de sua comunidade.

Para isto, salienta-se que mais estudos são necessários para aprimorar os recursos metodológicos de coletas de dados etnobotânicos específicos para crianças e adolescentes. Desta maneira, pode-se compreender o conhecimento nos diferentes níveis etários, sua geração e transmissão dentro da estrutura da unidade familiar.

\section{Referências}

ACOSTA, M.E.; VIGNALE, N.D.; LADIO, A.H. ¿Qué saben sobre plantas empleadas en medicina tradicional los niños de una escuela primaria de s. S. De jujuy, argentina? Gaia Scientia, v. 9, n.3, p. 90-104, 2015.

ALBUQUERQUE, U.P.; ANDRADE, L.H.C. Conhecimento botânico tradicional e conservação em uma área de caatinga no estado de Pernambuco, Nordeste do Brasil. Acta Botanica Brasilica, v. 16, n. 3, p. 273-285, 2002.

ALBUQUERQUE, U.P.; LUCENA, R.F.P.; CUNHA L.V.F.C. (eds). Métodos e técnicas na pesquisa Etnobiológica e Etnoecológica. Editora NUPEEA, Recife, v. 1, p. 53-54, 2010.

ALBUQUERQUE, U.P. Introdução à Etnobotânica. $2^{a}$ ed. Rio de Janeiro: Interciência, p. 120, 2005.

AMOROZO, M.C.M. Perspectiva Etnobotânica na Conservação de Biodiversidade. In: Congresso da Sociedade Botânica de São Paulo, XIV, Rio Claro: UNESP, p. 2, 2002.

BORTOLOTTO, I.M.; GUARIM-NETO, G. Conservação da natureza em uma escola rural do distrito de Albuquerque (Corumbá, Mato Grosso do Sul): uma abordagem para a educação no contexto da etnobotânica. Revista de Educação Pública, v. 7, n.11, p. 25-41, 1998.

EMPERAIRE, L.; VELTHEM, L.; OLIVEIRA, A.G. Patrimônio cultural imaterial e sistema agrícola: o manejo da diversidade agrícola no médio Rio Negro (AM), comunicação à 26a Reunião Brasileira de Antropologia, ABA. 0104/06/2008, Porto Seguro (BA), 2008.

FREITAS, A.V.L.; COELHO, M.F.B.; MAIA, S.S.S.; AZEVEDO, R.A.B. A percepção dos quintais rurais por crianças de São Miguel, Rio Grande do Norte, Brasil. Revista Verde, v. 6, n. 2, p. 212-220, 2011.

FREITAS, R.E.; RIBEIRO, K.C.C. Percepção ambiental com crianças da creche bom pastor, belo horizonte/mg: uma experiência de extensão, Revista Eletrônica Aboré- Manaus - Edição 03, 2007. 
GARCÍA, G.S.C. The mother-childnexus. Knowledge and valuation of wild food plants in Wayanad, Western Ghats, India Journal of Ethnobiology and Ethnomedicine, 2006.

GUERRA, N. M.; LEITE, A.P.; SOUZA, A.S.; RIBEIRO, J.E.S; RIBEIRO, J.P.O.; OLIVEIRA, R.S.; ALVES, C.A.B.; SOUSA JÚNIOR, S.P. LIMA, J.R.F.; LUCENA, R.F.P. Uso de algaroba (Prosopis juliflora (S .W.) DC ) en las comunidades tradicionales de las regiones semiáridas del Nordeste de Brasil. Gaia Scientia, v. Especial, p. 124-136, 2014.

IBGE. INSTITUTO BRASILEIRO DE GEOGRAFIA E ESTATÍSTICA. Disponível em: <https://www.ibge.gov.br/>. Acesso em: 23 maio. 2017.

ITURRA. R. O PROCESSO EDUCATIVO: ENSINO OU APRENDIZAGEM. In: Revista Educação, Sociedade \& Culturas, Afrontamento, Porto, n. 1, 1994.

LIMA, J. R. F.; ALVES, C. A. B.; RIBEIRO, J. E. S.; CRUZ, D. D.; MOURÃO, J. S.; TORRE CUADROS, M. L. A.; LUCENA, R. F. P. Uso e disponibilidade de espécies vegetais nativas no semiárido do Nordeste do Brasil: uma análise da hipótese da aparência ecológica. REDE - Revista Eletrônica do PRODEMA, v. 10, n. 1, p. 110-131, 2016. ISSN: 1982-5528.

LUCENA, C.M.; LUCENA R.F.P.; COSTA, G.M.; CARVALHO, T.K.N.; COSTA, G.G.S.; ALVES, R.R.N.; PEREIRA, D.D.; RIBEIRO, J.E.S.; ALVES, C.A.B.; QUIRINO, Z.G.M.; NUNES, E.N. Use and knowledge of Cactaceae in Northeastern Brazil. Journal of Ethnobiology and Ethnomedicine, v. 9, n. 62, 2013.

LUCENA, C.M.; CARVALHO, T.K.N.; RIBEIRO, J.E.S.; QUIRINO, Z.G.M.;

CASAS, A.; LUCENA, R.F.P. Conhecimento botânico tradicional sobre cactáceas no semiárido do Brasil. Gaia Scientia. Edição especial Cactaceae, v. 9, n. 2, p. 77-90, 2015.

MCCARTER, J.; GAVIN, M.C. Perceptions of the value of traditional ecologicalknowledge to formal school curricula: opportunities and challenges from Malekula Island, Vanuatu, Journal of Ethnobiology and Ethnomedicine, 2011.

LINS NETO, E.M.F; PERONI, N.; ALBUQUERQUE, U.P. Traditional knowledge and management of Umbu (Spondias tuberosa, Anacardiaceae): An endemic species from the semi-arid region of Northeastern Brazil. Economic Botany, v. 64, n. 1, p. 11-21, 2010.

NASCIMENTO, E. C. S.; TABARELLI, M.; SILVA, C.A.D.; LEAL, I.R.; TAVARES, W.S.; SERRÃO, J.E.Z.; ZANUNCIO, J.C. The introduced tree Prosopis juli flora is a serious threat to native species of the Brazilian Caatinga vegetation. Science of the Total Environment, v. 481, p. 108-113, 2014.

OLIVEIRA, F.C.; ALBUQUERQUE, U.P.; FONSECA-KRUEL, V.S.; HANAZAKI, N. Avanços nas pesquisas etnobotânicas no Brasil. Acta Botanica Brasilica, v. 23, n. 2, p. 590-605, 2009. 
PEREIRA, B.E.; DIEGUES, A.C. Conhecimento de populações tradicionais como possibilidade de conservação da natureza: uma reflexão sobre a perspectiva da etnoconservação. Desenvolvimento e Meio Ambiente, Editora UFPR, n. 22, p. 37-50, 2010.

QUINLAN, M. Considerations for collecting freelists in the field: examples from ethobotany. Field methods, v. 17, n. 3, p. 219-234, 2005.

SETALAPHRUK, C.; PRICE, L.L. Children's traditional ecological knowledge of wild food resources: a case study in a rural village in Northeast Thailand Journal of Ethnobiology and Ethnomedicine, v. 3, n. 33, 2007.

SILVA, N.; LUCENA, R. F. P.; LIMA, J. R. F.; LIMA, G. D. S.; CARVALHO, T. K. N; SOUSA JÚNIOR, S. P.; ALVES, C. A. B. Conhecimento e Uso da Vegetação Nativa da Caatinga em uma Comunidade Rural da Paraíba, Nordeste do Brasil. Boletim do Museu de Biologia Mello Leitão, v. 34, 5-37 p. 5-37, 2014.

SILVA, J.M.C.; LEAL, I.R.; TABARELLI, M. (ed.). Caatinga: The largest tropical dry forest region in South America. Springer International Publishing, 2017.

WYNDHAM, F.S. Environments of Learning: Rarámuri Children's Plant Knowledge and Experience of Schooling, Family, and Landscapes In The Sierra Tarahumara, Mexico, Springer Science + Business Media, Llc. 2009. 